\title{
MONBODDO Y ROUSSEAU (1933)
}

\author{
Arthur Lovejoy
}

\section{Traducido del inglés por Teresa Calderón Quindós Presentación por Fernando Calderón Quindós \\ Universidad de Valladolid quindos@fyl.uva.es}

\begin{abstract}
Resumen
El texto que aquí se presenta por vez primera en español es una exposición de las principales tesis sostenidas por Monboddo y Rousseau en torno a la naturaleza de los primates superiores. Este tema suscitó un prolongado y fecundo debate a lo largo del siglo XVIII, y dio lugar a las primeras intuiciones evolucionistas posteriormente desarrolladas por Charles Darwin.

Palabras clave: Monboddo, Rousseau, primates, estado de naturaleza, evolucionismo.
\end{abstract}

\section{Abstract}

The work-here published for the first time in Spanish translation-presents the main theses maintained by Monboddo and Rousseau regarding the nature of higher primates. This subject-matter motivated a long and deep discussion throughout the $18^{\text {th }}$ century, and triggered the first evolutionist intuitions later developed by Charles Darwin.

Keywords: Monboddo, Rousseau, primates, state of nature, evolutionism.

\section{Presentación ${ }^{*}$}

\section{$\overline{\mathrm{R} A}$}

El célebre naturalista sueco Carl Linné, decidido a clasificar la inmensa variedad fáunica conocida hasta mediado el siglo XVIII, incluyó al hombre y los primates en el género homo, reservando para aquél el nombre específico de homo sapiens. La anatomía comparada era aún en pleno siglo XVIII una ciencia escasamente fiable, y los

* Presentación de Fernando Calderón Quindós, investigador post-doctoral, Departamento de Filosofía, Universidad de Valladolid. 
primates superiores incluidos por Linné en el género homo eran auténticos desconocidos para el europeo. Tan solo algunos viajeros y un reducido número de curiosos y científicos habían tenido oportunidad de examinarlos, y ni los estudios eran tan exhaustivos, ni las condiciones y medios tan adecuadas. Prueba de ello es la utilización del término orang-outang, empleado por la ciencia europea del siglo XVIII para referirse tanto a los primates de las Indias Orientales como a sus parientes evolutivos del interior de África.

La propuesta de Linné, en todo caso, parecía precipitada, pues antes de ubicar al hombre junto a los primates en el género homo, era necesario determinar la naturaleza exacta de estos últimos, asunto que aún estaba por decidir. Para el naturalista francés Buffon o el médico inglés Tyson no había duda de que los seres antropomorfos no eran hombres, sino bestias. En cambio, el filósofo escocés James Burnet, conocido por sus contemporáneos como lord Monboddo, representaba la opinión contraria. El primer volumen de su obra Origin and Progress of Language (1774) y algunas de las cartas incluidas en su correspondencia personal contienen una amplia y rigurosa defensa de la humanidad de los orangutanes, primates cuyo nombre de origen malayo significa precisamente "hombre que habita las selvas". La posición intermedia entre Buffon y Tyson, por un lado, y Monboddo, por otro, la representan tres autores franceses: Maupertuis, La Mettrie y Rousseau. Los argumentos que Buffon y Tyson habían alegado como pruebas concluyentes para negar a los primates su condición de hombres, fueron contestados por estos últimos con pruebas contrarias, suficientes al menos para alertar del riesgo de una respuesta rotunda. Un fragmento de las Lettres de Maupertuis, escritas en 1752, ilustra ejemplarmente esta actitud de cautela. Refiriéndose a los primates de África escribe: "Puedo concluir quizás que no están hechos para vivir en sociedad conmigo, pero no debo concluir por ello que no tengan alma [...]. Paso del mono al perro, al zorro, y por grados imperfectos desciendo hasta la ostra, y quizás hasta la planta, la cual no es sino una especie de animal más inmóvil aún que la ostra, sin que haya razón ninguna para detenerme en punto alguno (Maupertuis, Oeuvres complètes, Lettres, Lyon, 1756, t. II, pp. 218-219).

El texto que aquí se presenta, "Monboddo y Rousseau", escrito por el filósofo norteamericano Arthur O. Lovejoy en 1933, recrea para el lector contemporáneo la discusión dieciochesca sobre la condición de los primates. Para ello, el autor se sirve de la opinión de los dos autores que dan nombre a su artículo. Rousseau anticipó en la nota X de su Discours sur l'origine de l'inégalité la opinión posteriormente defendida por Monboddo. El filósofo de Ginebra había sugerido la posibilidad de que los seres antropomorfos descubiertos por los viajeros europeos pudieran ser hombres en estado de naturaleza, y el magistrado escocés lord Monboddo proporcionó una justificación científica detallada al atrevimiento especulativo de su contemporáneo.

Sin duda alguna, el asunto de los primates fue uno de los temas de discusión favoritos entre los intelectuales del siglo XVIII. La demostración de ello la encontramos en un breve pasaje de The Life of Samuel Johnson, escrito por James Boswell en 1791. En dicho pasaje, Boswell reproduce una entrevista mantenida por él con el moralista inglés Johnson sobre el asunto de los orangutanes, entrevista en la que las opiniones 
de Monboddo y Rousseau son discutidas y diferentemente valoradas. Lovejoy emplea este pasaje como introducción literaria a su artículo.

La decisión de traducir "Monboddo y Rousseau" por vez primera al español responde básicamente a dos razones: i) porque Lovejoy rescata del olvido filosófico la figura del magistrado escocés Lord Monboddo, al que reconocerá el mérito de haber anticipado las tesis evolucionistas expuestas por Darwin en The origin of species, y ii) porque Lovejoy rescata igualmente la discusión dieciochesca sobre la condición de los primates superiores, discusión superada en la actualidad, pero de la que aún falta por resolver su capítulo moral. En el siglo XVIII, en efecto, se trataba de determinar si los monos antropomorfos de África y las islas de Borneo y Sumatra eran o no hombres. Resuelta esta cuestión, se trata ahora de determinar si estos mismos seres merecen el rango de personas, en cuyo caso habría de serles reconocida la titularidad de ciertos derechos.

\section{Nota sobre la traducción}

La traducción al español sigue el texto siguiente: "Monboddo and Rousseau", en Arthur Lovejoy, Essays in the History of Ideas, Baltimore: The John Hopkins Press, 1948, cap. III, pp. 38-61. Lovejoy, no obstante, incluyó este artículo en una publicación anterior de 1933, cuyos datos de edición figuran en la nota 1 de esta traducción. 


\section{Monboddo y Rousseau ${ }^{1}$}

El treinta de septiembre de 1769, Boswell y su héroe comían en el Mitre. Durante algún tiempo, y a intervalos, Boswell había sentido la tentación de volver al estado de naturaleza. Pocas fueron las epidemias o enfermedades intelectuales que no padeció. Como han demostrado los estudios de Miss Lois Whitney, parece que en los años 50 y 60 ciertas formas de primitivismo habían sido celebradas en la región natal de Boswell con mayor entusiasmo que en las porciones meridionales de la isla ${ }^{2}$, y aunque su anterior adoración por Rousseau estaba ya abatida, algunos de sus efectos perduraban. En cualquier caso, casi ningún tema podría haber sido más apropiado para aquello que Boswell consideraba el fin último de la vida cuando estaba en Londres: encontrar asuntos de conversación suficientemente estimulantes como para provocar en el Gran $\mathrm{Oso}^{3}$ uno de sus mejores arranques. Boswell, por tanto, en esta ocasión, tal como nos dice, "intentaba argumentar a favor de la felicidad superior de la vida salvaje, frente a los temas caprichosos y ordinarios".

-JOHNSON. "Señor, no puede haber nada más falso que eso. Los salvajes no tienen ninguna ventaja corporal de la que no disfruten los hombres civilizados. No tienen mejor salud; y en cuanto a la inquietud y al desasosiego mentales, no están por encima sino por debajo, como los osos. No, Señor, no diga usted tal paradoja, no quiero oír más sobre ello. Ni aquello puede entretener, ni mucho menos instruir. Lord Monboddo, uno de vuestros jueces escoceses, hablaba mucho de tal desatino. Lo toleré de él; pero no voy a tolerarlo de vos".

-BOSWELL. "Pero, Señor. ¿No afirma Rousseau tal desatino?"

-JOHNSON. "Es cierto, Señor, pero Rousseau sabe que lo que está diciendo es un desatino, y se ríe de que el mundo lo mire asombrado".

-BOSWELL. "¿Cómo es eso, Señor? Pero, Señor, un hombre que dice desatinos tan bien, debe saber que está diciendo desatinos. Sin embargo me temo (sonriendo, socarrón) que Monboddo no sabe que está diciéndolos..." ${ }^{4}$

Publicado por primera vez en Modern Philology, XXX (1933), pp. 275-296 (N. del T.).

"English Primitivistic Theories of Epic Origins", Mod. Phil., XXI (1924) pp. 337-78. Para una ilustración de Boswell en un estado primitivista, cfr. Letters of James Boswell, ed. Tinker (1924), I, p. 98 (Febrero 1, 1767): "Estás tentado a unirte a Rousseau en su preferencia por el estado salvaje. Yo también lo estoy a veces. Cuando estoy cansado de los negocios, o atormentado por las pasiones de la vida civilizada, huiría al bosque“, etc.

Apodo con el que James Boswell se refiere a su biografiado Samuel Johnson (N. del T.).

Boswell's Life of Johnson, ed. G. B. Hill Oxford, 1887, II, pp. 73-74. Para conocer la perspectiva del Dr. Johnson con respecto a la vida salvaje, cfr. también el Adventurer, n. ${ }^{\circ} 67$ (1753). Por otro lado, en el Rambler, n. ${ }^{\circ} 33$ (1750), Johnson había dibujado un cuadro de la Edad de Oro, desaparecida una vez que el hombre comenzó a desear la propiedad privada. "Luego llegaron la violencia y el fraude, el robo y la rapiña. Poco después el orgullo y la envidia irrumpieron en el mundo; y trajeron consigo una nueva jerarquía de valores. Los hombres, que hasta entonces creíanse ricos por no tener necesidad de nada, tasaban ahora sus demandas ya no en función de su valor natural, sino en función de la abundancia de los otros; 
No es necesario añadir que Boswell no estaba del todo confundido, ni siquiera en este último punto. A continuación, señala que él mismo, en ocasiones, había "sentido el deseo de retirarse a un desierto". Pocas sentencias hay más johnsonianas que la réplica suscitada por esta actitud: "Señor, ya tiene suficiente desierto en Escocia"

Cuando, más tarde, Johnson y Boswell comenzaron a charlar sobre lo que hoy llamamos "primitivismo", los dos contemporáneos que primero vinieron a sus mentes como representantes de esa "paradoja" fueron el autor de los dos Discours de Dijon y un noble escocés políticamente activo cuyas teorías no serían hechas publicas al mundo hasta cuatro años más tarde, pero de las que, no obstante, se tenía conocimiento en los círculos frecuentados por él en Edimburgo y Londres. La diferencia entre los dos, en opinión de Johnson, era simplemente que el escocés era un primitivista sincero y el francés uno insincero.

La verdad, sin embargo, era que, aunque las ideas de ambos estaban equivocadas, no estaban faltas de todo fundamento, y el actual estudiante de historia de las ideas que se limita a señalar a Rousseau y Monboddo como simples "primitivistas" pierde de vista lo realmente importante e interesante de sus posturas. Que ambos a veces se deleitaron en el gozo de la vida salvaje resulta innegable. Monboddo -o James Burnet, como se llamaba antes de su nombramiento como magistrado- había ingresado en el Marischal College de la Universidad de Aberdeen en 1728, a la edad de

y comenzaron a considerarse a sí mismos pobres, cuando veían que las posesiones de sus vecinos excedían las propias". En esta versión de la Edad de Oro, sin embargo, "Rest" toma el lugar de la Astrea. Johnson acababa de anticipar en este pasaje la vena primitivesca y comunista de los discursos de Rousseau. Pero, por supuesto, esto no es en absoluto sorprendente, ya que el introito del ensayo era simplemente una nueva miscelánea de fragmentos de Arato, Ovidio y Séneca, mientras que los discursos de Rousseau, en cuanto que primitivistas, eran en gran medida simples variaciones sobre los mismos temas clásicos

5 Monboddo no se tomaba bien las bromas de Johnson. En su Origin and Progress of Language, $2^{\mathrm{a}}$ ed., 1789 , pp.: 262-275 (en lo sucesivo citado como $O$. and P.) aborda el gusto, el aprendizaje e incluso el carácter del Gran Sam con verdadero odio. Johnson "no era ni un estudioso ni un hombre con buen gusto"; no era "la vigésima parte de la décima parte de un crítico"; era "el hombre más envidioso y maligno que yo jamás haya conocido, que no elogiaba ni autor ni libro que otra gente elogiara, y en la conversación privada siempre estaba dispuesto a criticar sin motivo y a contradecir cualquier cosa que fuera dicha, y no admitía con paciencia que otras personas fueran el centro de atención por una sola vez". El trasfondo explícito de este arrebato, no obstante, no residía en los ataques de Johnson sobre Monboddo (de los que Boswell había informado a su paisano) sino, por un lado, en los menosprecios de Johnson hacia el estilo de Milton en inglés y en latín, y por otro, en su afirmación de que "Paradise Lost no es uno de los más grandes poemas heroicos, solo porque no es el primero". En opinión de Monboddo, "el tema de Paradise Lost es demasiado elevado para imitarse poéticamente; mientras que la historia de La Ilíada de Homero es el mejor tema para cualquier poema épico que jamás se haya inventado o, hablando con propiedad, que jamás se haya elegido". Algunos de los reproches de Monboddo sobre la crítica literaria de Johnson y su erudición no son gratuitos. La estima mutua manifestada por ellos, como anfitrión y huésped, durante la visita de Johnson a Monboddo en 1773 -uno de los pasajes más atractivos de la obra de Boswell Tour to the Hebrides- evidentemente no duró. 
catorce años, sintiendo muy pronto la influencia de Thomas Blackwell el joven. Algunas de las ideas favoritas de los primitivistas de Aberdeen, en las que había sido adoctrinado a tan tierna edad, continuaron influyendo en sus opiniones sobre ciertos asuntos a lo largo de su vida. Sin embargo, su principal importancia en la historia intelectual de Gran Bretaña no se debe a su labor de portavoz del primitivismo, sino a haberse erigido en uno de los iniciadores (en ese país) de una nueva forma de pensar contraria al primitivismo. Y en esto su posición es paralela a la de Rousseau -al menos al del Rousseau del Discours sur l'origine de l'ìnégalité. Ya he demostrado en el ensayo anterior que lo comparativamente original, innovador e históricamente trascendental en ese discurso era precisamente lo contrario de lo que muchos de los historiadores de la literatura francesa y de la filosofía han considerado. Aunque en el Second Discours aún sobrevivía mucho de la dilatada tradición primitivista, el rasgo que permitió a su autor introducir una nueva fase en la historia del pensamiento consistía en cierto tipo de evolucionismo social; y el evolucionismo es, en esencia, por supuesto, el extremo contrario del primitivismo, aunque a mediados del siglo XVIII los dos aparecían formando varias combinaciones curiosas. En este trabajo demostraré que lo que he observado previamente con respecto a Rousseau es también cierto en Monboddo ${ }^{6}$. Plantearé, de manera incidental, la cuestión de si la semejanza entre sus ideas puede atribuirse a la influencia de Rousseau sobre el escritor escocés, e indicaré las razones por las que pienso que Monboddo todavía fue más lejos que Rousseau en este sentido.

En la obra más importante de Monboddo, como en el Discours de Rousseau, vamos a encontrar las seis tesis siguientes, todas ellas inusuales, y algunas de ellas importantes novedades en el tercer cuarto del siglo XVIII.

1. Que el estado de naturaleza, o condición original de la humanidad, era una condición de pura animalidad, en la que nuestros ancestros carecían de lenguaje, de organización social y de casi todas las destrezas prácticas, y en la que, en general, no se distinguían de los simios por sus logros intelectuales o por su modo de vida.

2. Que, por tanto, el estado de naturaleza, denominado propiamente así, no era un estado ideal, excepto con respecto a la condición física del animal humano. Era una fase de la cual era no solo inevitable sino también deseable que la humanidad emergiera. En este punto, sin embargo, las afirmaciones tanto de Rousseau como de Monboddo no están del todo libres de inconsistencias; y ambos, aunque sin ser verdaderos primitivistas, pueden llamarse "retrospectivistas". Uno y otro vieron el mejor capítulo de la historia humana en una fase anterior del desarrollo del hombre, pero no en la primera fase: Rousseau la encontró en el estadio campesino de la evolución cultural y Monboddo en la antigua Grecia.

3. Que el hombre y el "orang-outang" son de la misma especie; en otras palabras, que los orangutanes son una porción de la raza humana que, por alguna razón, no ha 
logrado desarrollarse como el resto; y que, por tanto, podemos ver en estos animales ejemplos aproximados de las características de nuestros más tempranos ancestros y de su modo de vida.

4. Que, como las anteriores proposiciones sugieren, la diferencia psicológica principal de la especie humana consiste no en atributos mentales o poderes reconocibles en la humanidad a lo largo de su historia, y por tanto presentes desde el comienzo, sino únicamente en la capacidad para el desarrollo gradual de facultades intelectuales más elevadas, lo que Turgot y Rousseau llamaron perfectibilité. Así, la historia del hombre comienza en un estadio en el que, en cierto sentido, no era todavía humano, en el que se diferenciaba esencialmente de otros animales solo por una latente capacidad de progreso. Solo cuando emergió del estado de naturaleza comenzó a ser un hombre verdadero.

5. Que, por tanto, la historia humana -al menos hasta un cierto punto-debería considerarse, no como se ha considerado comúnmente, como un proceso de declive desde una primitiva perfección a un oscurecimiento gradual de la luz pura de la naturaleza que al principio había iluminado a los hombres, sino más bien como un ascenso lento y doloroso desde la salvaje animalidad a la vida de un ser racional y social.

6. Que, en consecuencia, se necesitaba una nueva historia de la ciencia que descubriera los sucesivos estadios de este proceso de desarrollo intelectual y evolución social, y que, para este propósito se requería principalmente un estudio mucho más profundo que ninguno que se hubiera hecho antes sobre la vida de los salvajes coetáneos, es decir, de las razas que todavía permanecen en uno u otro de los estadios culturales típicos que se supone han atravesado los antecesores de los pueblos civilizados.

He demostrado anteriormente que todas estas tesis se pueden rastrear en el Second Discours de Rousseau; ahora demostraré que estas tesis se encuentran también en los escritos de Monboddo, principalmente en el primer volumen de su Origin and Progress of Language (1773), y en algunas de sus cartas publicadas en el volumen del profesor Knight, Lord Monboddo and Some of His Contemporaries (1900).

\section{Carácter del estado de naturaleza}

Es un hecho establecido -dice Monboddo-

que ha habido en el mundo, y todavía hay, rebaños de hombres (pues no merecen el nombre de naciones) viviendo en un estado enteramente embrutecido, y de hecho, y desde varios puntos de vista, más salvajes que algunas bestias, puesto que no tienen ni gobierno ni cultura [...]. Donde quiera que haya progreso, debe haber un comienzo, y el comienzo en este caso no puede ser otro que el de mero animal. Y si retrocedemos por la ruta del progreso, ¿dónde debemos parar? Habiendo descubierto tantos eslabones de la cadena, tenemos libertad para suponer el resto, y concluir que el comienzo 
debe remontarse a esa naturaleza común que nos conecta con el resto de la creación animal. La observación de los hombres salvajes nos conduce naturalmente a considerar la condición de las bestias. Entre éstas y los salvajes hay tales parecidos que muchos apenas admiten diferencia alguna; e incluso entre nosotros y las bestias no hay ninguna diferencia física ni en nuestro nacimiento, ni durante algún tiempo considerable posterior a nuestro nacimiento ${ }^{7}$.

Monboddo escribió de modo similar a su amigo James Harris, el autor de Hermes, en 1772:

Creo que muchos han pensado que he desprestigiado demasiado nuestra naturaleza. Porque si bien nadie tiene una idea más elevada que yo de la naturaleza humana, siempre que haya sido mejorada por las artes de la vida y exaltada por la ciencia y la filosofía, no puedo concebir que -antes de la invención del lenguaje- se haya encontrado el hombre en un estado superior al de la bestia. En resumen el mutum ac turpe pecus de Horacio es mi noción del hombre en su estado natural y original; y para fundamentar mi filosofía, he apelado a la historia -tanto antigua como moderna- en busca de pruebas de la tosca condición en la que muchas naciones han sido descubiertas antes de que pudieran usar el lenguaje de algún modo. De lo cual podemos inferir cuán abyecta y embrutecida debe haber sido su condición antes de que tuvieran la facultad del habla ${ }^{8}$.

\section{Indeseabilidad del estado de naturaleza}

Obviamente es inconcebible que Monboddo se haya lamentado de la emergencia del hombre desde esta "condición abyecta y embrutecida", o haya deseado que la raza volviera a ella. Por el contrario, nos ha dicho expresamente que la naturaleza humana alcanza su estado más elevado solo "cuando ha sido mejorada por las artes de la vida y exaltada por la ciencia y la filosofía". No obstante, hay en Monboddo, como en Rousseau, pasajes que podrían fácilmente considerarse elogios de este estado de naturaleza descrito por ambos en términos tan seductores. Con respecto a Rousseau, la aparente incongruencia entre estas dos posiciones fue señalada por Voltaire: "Para razonar consecuentemente, todo enemigo del lujo debe creer con Rousseau que el estado de felicidad y de virtud del hombre no es aquél del salvaje, sino el del orangu-

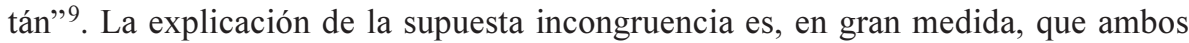
escritores, al alabar la "condición natural de la humanidad", se referían fundamentalmente a la superioridad anatómica del bruto primitivo, y lamentaban el deterioro físico de nuestra especie, achacable, según ellos, a los lujos de la sociedad civilizada. Así Monboddo:

O. and P. 2. ${ }^{\mathrm{a}}$ ed. (1774), I, p. 147.

Knight, op. cit., p. 73.

Dict. philos., art. "Luxe". 
Si es verdad, como creo firmemente, que el estado en el que Dios y la Naturaleza han colocado al hombre es el mejor, al menos en lo que respecta a su cuerpo, y que ninguna habilidad puede provocar mejora alguna en el hábito y la constitución natural del cuerpo humano, creo entonces que conocer este estado natural debe ser de la mayor importancia, y lo más útil en la práctica de las diferentes artes y en el modo de vida en general. El objeto, por ejemplo, del arte de un médico debe ser devolver el cuerpo a ese estado natural, en la medida de lo posible, estado natural que debe ser por tanto el modelo de perfección de su arte. El filósofo político, de modo similar, estudiará cómo preservar la fuerza natural y el vigor del animal [...] mediante una dieta apropiada, ejercicio, y hábitos de vida determinados [...]. Y finalmente, cada hombre en particular [...] si es sabio y si conoce este estado de naturaleza, intentará devolverse a sí mismo a tal estado, en la medida en que sea coherente con el estado de sociedad en el que vive; y, siguiendo el ejemplo de los grandes hombres de la antigüedad, sufrirá por elección propia esos tormentos, comúnmente así considerados, que el salvaje sólo sufre por necesidad, sin saber que son absolutamente necesarios para su felicidad ${ }^{10}$.

Los pasajes de Monboddo sobre las ventajas del estado de naturaleza eran entonces una manera de expresar un ideal extraño en su época y que, sin duda, necesitaba enorme propaganda: el de la buena salud y forma física. Vitupera la "constante falta de moderación en el comer y en el beber" de sus coetáneos, y lamenta que "los ejercicios atléticos, al menos aquellos que son apropiados para potenciar la fuerza y agilidad del cuerpo, estén casi en entero desuso"11. Era, en resumen, un temprano y celoso, aunque inefectivo, profeta de la cultura física y un predicador del valor higiénico en un régimen casi espartano ${ }^{12}$. Sus elogios ocasionales del estado de naturaleza deben entenderse en parte como vestigios de una antigua convención, si bien que inspirados paradójica y principalmente por el loable objetivo de mejorar la condición física de la humanidad dieciochesca. Pero del naturalismo ético, vinculado frecuentemente al primitivismo y a la filosofía general que ello implicaba, Monboddo era más adversario que abogado defensor. Creía en la degradante influencia de las artes y las ciencias solo en cuanto que potencian el lujo y el ablandamiento físico; $y$, en contra de lo que opinaba Rousseau, no pensaba que esto fuera una consecuencia inevitable del

$10 O$. and P., I, iii; también: "El filósofo político [...] estudiará el modo de preservar la fuerza y el vigor natural del animal (las destrezas humanas pueden conseguirlo), mediante ejercicio y hábitos de vida apropiados, y cómo prevenir, en la medida de lo posible, la indulgencia de la comodidad y del placer carnal, por la cual la raza de los hombres civilizados de todas las naciones ha sufrido un declive desde los tiempos más remotos".

11 Ibíd., III, p. 453; cfr. también I, p. 447 n.: "Sin duda es cierto el hecho de que el hombre es actualmente más propenso a la enfermedad que cualquier otro animal; pero la culpa debe buscarse donde realmente reside, en los malos modos e instituciones, y en las muchas e ingeniosas artes que hemos inventado para la destrucción de nuestros cuerpos, no en Dios y en la naturaleza".

12 La necesidad de tal predicación en Escocia en este periodo se ilustra en la obra de Dean Ramsay, Reminiscences of Scottish Life and Character, 1885. 
cultivo de las mismas. Por el contrario, mantenía que "nuestras ventajas sobre los salvajes dependen únicamente de nuestras artes y ciencias"13.

No puede haber lo que apropiadamente llamamos virtud, mientras el hombre no se transforme en un animal racional y político. Es sólo entonces cuando aquél exhibe su verdadero coraje, muy diferente de la ferocidad de la bestia o del salvaje; su generosidad, su magnánimo desprecio por el peligro y por la muerte, su amistad y amor hacia su propia nación acompañadas de las otras virtudes que tanto ensalzan a la naturaleza humana, pero que apenas podemos esperar encontrar en el simple salvaje o en la bestia o en los infantes de nuestra especie ${ }^{14}$.

\section{El hombre y el orangután}

Rousseau había marginado su sugerencia sobre nuestro parecido con los simios a una nota. Allí, sin duda, escapó a la atención de muchos de los lectores de su propio tiempo, y pasó aparentemente inadvertida para muchos de los historiadores posteriores. Monboddo, sin embargo, dedicó más de cien páginas a la defensa de esta hipótesis, y probablemente fue con esta doctrina con la que fundamentalmente se asoció su nombre en las mentes de la mayor parte de sus coetáneos después de que en 1773 apareciera su primer volumen de Origin and Progress ${ }^{15}$. Aquí había un tema más sabroso todavía para las mofas de Johnson que las nociones de Monboddo sobre los salvajes: "Señor, es tan posible que el Orangután no hable como que hable. Sin embargo, no lo discutiré. Yo nunca habría pensado que pudiera encontrar un Monboddo; y sin embargo él existe". "Es una pena", dijo Johnson de nuevo, "ver a Monboddo publicar esas ideas tal como lo ha hecho; un hombre sensato, y un erudito tan elegante. No habría tenido ninguna importancia de haberlo hecho un loco, simplemente reiríamos, pero cuando lo hace un hombre sabio, nos apenamos. Hay otra gente que tiene ideas extrañas, pero las oculta; sin embargo, Monboddo es tan celoso de sus ideas como una ardilla de su cola". Con gran dureza la historia de la ciencia y de la filosofía en el siglo siguiente volvería contra el mismo Dr. Johnson su burla mordaz.

Transcurrido en torno a un mes de la publicación del primer volumen de Origin and Progress, Johnson le dijo a Boswell: "Señor, todo es conjetura sobre una cosa inútil, aun cuando se llegue a saber que es cierta. El conocimiento de todo género es

15 Rousseau y Monboddo son los "escritores recientes" a los que se refiere el "fundador de la antropología", J. F. Blumenbach, en su Tesis Doctoral, De generis humani varietate nativa (1775), y de los que dice que "no se enrojecieron al avanzar la doctrina de la similitud del hombre con el orangután", doctrina que Blumenbach trata aquí con arrogancia al decir que "no necesita ninguna refutación apud rei peritos". Una versión inglesa de esto puede encontrarse en Blumenbach's Antropological Treatises (1865), p. 95, trad. T. Bendyshe. 
bueno. La conjetura sobre cosas útiles es buena; pero conjeturar sobre algo cuyo conocimiento es inútil, como si el hombre caminaba a cuatro patas, es muy improductiva" ${ }^{16}$. Johnson era también hombre de una sensatez fuera de lo común y un elegantísimo erudito, pero esa frase fue quizás la cosa más profundamente estúpida dicha por un hombre de su generación. Rechazar lo que Boswell llama "las especulaciones extrañas de Lord Monboddo sobre el estado primitivo de la naturaleza humana" no era sino expresión de un conservadurismo natural, tal como debía esperarse de un hombre del tiempo y temperamento de Johnson; pero declarar que la cuestión surgida era de importancia nula e improductiva suponía dar muestras de una extraña ceguera hacia la trascendencia de las ideas, así como de una singular carencia de imaginación científica y filosófica. ¡Cómo brilla en cambio Monboddo en la conclusión de sus dos capítulos sobre el orangután!, escrita con la moderación de un espíritu científico.

No osaré decir que mis hechos y argumentos sean tan convincentes como para no dejar duda de la humanidad del orangután, pero me aventuraré a afirmar que he dicho suficiente para que el filósofo considere este asunto con alguna circunspección, y lo convierta en materia digna de ser investiga$\mathrm{da}^{17}$.

Aparentemente, para Monboddo el término "orangután" era genérico, aplicable también al gorila y al chimpancé. De hecho, normalmente se empleaba para referirse a los simios africanos más que a los orangutanes de Borneo o Sumatra. Su primera razón para asegurar nuestra probable consanguinidad con estos antropoides era, en cierta medida, de tipo científico y completamente legítima, basada en los resultados de anatomía comparada recientemente expuestos por Buffon y Daubenton en la Histoire naturelle. Según éstos parecía, dice Monboddo, que

en cuanto a su cuerpo, (el orangután) es en conjunto un hombre, tanto por fuera como por dentro, exceptuando algunas variaciones tan pequeñas que no es posible establecer sobre ellas diferencias específicas entre los dos animales, y estoy persuadido de que éstas son menos considerables que las que podemos encontrar entre individuos que sin duda pertenecen a la raza humana. Y más en particular, dice el Sr. Buffon, su lengua y los otros órganos de pronunciación son los mismos que los del hombre. Ambos tienen las mismas vísceras [...] exactamente con la misma estructura, y sólo ellos tienen glúteos y pantorrillas, lo que les permite caminar más erguidos que cualquier otro animal ${ }^{18}$.

16 Boswell's Life of Johnson, ed. Hill, V, 46, III; II, pp. 259-60 (Mayo 8, 1773).

17 O. and P., I, p. 360.

18 Ibíd.,p. 271. Aparentemente, Monboddo también conocía la obra de Edward Tyson Orangoutang, sive homo sylvestris; or, the anatomy of a Pygmie compared with that of a Monkey, an Ape, and a Man (1699), primer estudio competente de la anatomía de un chimpancé; y la descripción de Tyson de los órganos del habla de tal animal era, para Monboddo, una evidencia importante a favor de esta teoría; cfr. el admirable trabajo de Ashley Montagu sobre el anatomista inglés, Edward Tyson (1943), p. 270. Tyson aportó una revisión exhaustiva de las 
No obstante, Monboddo estaba más interesado en el "principio interior" del orangután, en los "hábitos y disposición mental", que en la anatomía del animal; y fue de éstos, más aún que de las similitudes de la estructura física, de donde extrajo la evidencia de la identidad específica entre los simios y nosotros mismos. En cuanto a los modos y costumbres del gorila y el chimpancé, recogió con esmero evidencias de muchas fuentes, y dio a algunos testigos mayor crédito del que debería esperarse de un experimentado abogado y juez escocés. A partir de todas las descripciones recogidas concluye:

La conclusión y esencia de todas estas relaciones es que el Orangután es un animal de forma humana, tanto por dentro como por fuera; que tiene inteligencia humana, tanta como puede esperarse de un animal que vive sin urbanidad y sin artes; que tiene una disposición mental mansa, dócil y humana; que tiene sentimientos y afectos comunes a nuestra especie, tales como el sentido de la modestia, del honor, y de la justicia, y del mismo modo y en ocasiones un vínculo tan fuerte de amor y amistad hacia otro individuo que un amigo no sobreviviría al otro; que viven en sociedad y tienen algunas destrezas, puesto que construyen cabañas y usan armas artificiales para atacar y defenderse, como palos, lo que ninguna simple bestia es capaz de hacer [...]. Del mismo modo, parece que muestran algún tipo de cortesía entre ellos, que practican ciertos ritos, como el enterramiento de los difuntos. Hechos como éstos son los que nos permiten preguntarnos si el Orang Outang pertenece o no a nuestra especie. El señor Buffon ha decidido que no. El señor Rousseau se inclina por la opinión contraria. Parece que el primero es consciente del peso de los hechos en relación con sus opiniones [...]. Algunos de nuestros naturalistas forman sus sistemas al margen de los hechos, $\mathrm{y}$ ajustan luego los hechos a sus prejuicios, considerando sólo de entre los primeros aquéllos que se adaptan mejor a sus propósitos y descartando todos los demás. Incluyo entre estos naturalistas al señor Buffon, cuya definición del hombre convierte la facultad de hablar en parte de su esencia y naturaleza, de lo que concluye que el puro estado de naturaleza, en el que el hombre no habría hecho uso de la palabra, es un estado absolutamente ideal e imaginario, y tal que no tuvo nunca una existencia real ${ }^{19}$.

descripciones previas de los simios antropoides que sin duda le fue útil a Monboddo. Tyson creía fervientemente en la continuidad de la cadena de los seres y, por tanto, se aproximaba, sin de hecho llegar a ella, a la conclusión de Monboddo: "en esta Cadena de la Creación, como vínculo intermedio entre el simio y el hombre colocaría a nuestro Pigmeo", es decir, el chimpancé. "El animal cuya anatomía he ofrecido parece ser el nexo de unión entre el Animal y el Racional". Sin embargo, Tyson afirma finalmente que "es un auténtico animal". (Orang-outang, pp. iii, p. 5). El parecido anatómico entre el hombre y el chimpancé era, por supuesto, inferior a lo supuesto comúnmente en la época de Monboddo, y no justificaba la teoría de la identidad de las "especies".

$19 O$. and $P$., I, pp. 289-93. Probablemente, Monboddo se refiere aquí principalmente al pasaje de Buffon sobre este asunto en Histoire naturelle, Tomo XIV (1766); cfr. especialmente pp. 3-4, 30-33, 37-38, 41-42. La conclusión de Buffon es: "Confieso que si tuviéramos que juzgar únicamente por la forma, la especie del mono podría ser tomada por una variedad de la especie humana". Sin embargo, "cualquiera que sea el parecido entre el hotentote y el mono, 
Monboddo ha sido ridiculizado con frecuencia, en su propio tiempo y desde entonces, por su conmovedora descripción del cortés gorila y el civilizado chimpancé, y apenas se puede negar que de algún modo no hubiera formado una idea demasiado exaltada de la faceta intelectual y del atractivo temperamento de nuestros primos los simios. Sobre el refinamiento de la hembra de la especie, en particular, cita algunos ejemplos bastante sorprendentes de "Bontius, el médico de Batavia" ${ }^{20}$, y de otros. A juzgar por tales ejemplos, parecería que los simios del sexo débil son modestos hasta el punto de la cursilería, y que están dotados de una sensibilidad de algún modo excesiva. Sin embargo, y aunque Monboddo exageraba, se encontraba más cerca de la realidad que muchos escritores posteriores hasta tiempos muy recientes. Después de siglo y medio de escepticismo científico, ahora se ha demostrado que los antropoides superiores poseen realmente algunas de las dotes que les atribuyó. Köhler ha probado que los chimpancés no son solo hábiles en el uso de utensilios, sino que también los fabrican; y los estudios cuidadosos de Yerkes y Kearton han mostrado los rasgos "casi humanos" de estos simios ${ }^{21}$

Son sin embargo tan considerables los logros atribuidos al orangután por algunos observadores a quienes Monboddo cita, que, desde su punto de vista, resultaba inequívoca la conclusión de que el orangután no representa la condición primitiva de

el intervalo que los separa es inmenso, ya que aquél conserva el pensamiento en el interior, y exhibe la palabra en el exterior". Monboddo busca eliminar este doble "intervalo", asegurando que el orangután posee una inteligencia bastante considerable, y argumentando que el hombre carecía de habla en el origen. Sobre las variaciones en los puntos de vista de Buffon respecto de la teoría de la evolución orgánica en general, véase la obra de Lovejoy, "Buffon and the Problem of Species", Popular Science Monthly, LXXIX (1911), pp. 464-473 y 554567.

20 Jakob de Bondt (Bontius), cuya Historia Naturalis et Medica Indiae Orientalis (1658) contenía un capítulo (1. V, cáp. 32) sobre el orang-outang sive homo silvestris, que concluía nihil ei humani deesse praeter loquelam. El simio de Bontius no era el chimpancé, sino el orangután de Borneo y Sumatra, donde había residido durante algunos años. (Batavia es el nombre con el que la Compañía Holandesa de las Indias Orientales bautizó a la actual Yakarta -N. del T.).

21 Köhler, The Mentality of Apes (1924); Koffka, The Growth of the Mind (1924), cap. IV; Yerkes, Almost Human (1925); C. Kearton, My Friend Toto (1925). Algunas de las observaciones de Yerkes pueden citarse por su cercanía a varios de los pasajes de Monboddo: "Se ha demostrado una y otra vez en relación con los tests de inteligencia que el orangután, el chimpancé y el gorila pueden utilizar y utilizan objetos de manera efectiva para alcanzar ciertos fines deseados como alimentos, la libertad y la oportunidad de jugar. Los resultados de los experimentos indican un orden de inteligencia que ciertamente sugiere la del humano, si no se acercan mucho a ella [...]. Los primates exhiben de diversas maneras los principales tipos de emoción que se dan en el hombre [...]. No es en absoluto sorprendente que los científicos tengan la impresión de que el chimpancé está más cerca del ser humano en su vida emocional que en ningún otro aspecto [...]. Este dibujo del tierno aspecto de la vida emocional de los monos y los grandes simios puede dar al lector una razón para detenerse y reflexionar. ¿Somos los humanos después de todo tan excepcionales por nuestro ostentoso altruismo?" Toto, el joven chimpancé de Kearton, era apenas menos "humano" que los "orangutanes" de Monboddo. 
la humanidad, sino un estado más avanzado. Así lo expuso Monboddo, y también lo hizo Rousseau con anterioridad a él. Monboddo mantiene, junto con Rousseau, que en el estado puro de naturaleza el hombre era una "bestia salvaje solitaria" sin "ninguna inclinación natural a entrar en sociedad", y que, por tanto, no vivía ni en rebaños ni en grupos familiares. Pero los orangutanes, según los informadores de Monboddo, algunas veces

viven juntos en sociedad; actúan de consuno, particularmente en el ataque a los elefantes; construyen cabañas, y sin duda practican otras artes para el sustento y la defensa [...]: se puede reconocer que están en el primer estadio del progreso humano, ya que están asociados y practican algunas habilidades vitales; pero no tan avanzados como para haber inventado el gran arte del lenguaje ${ }^{22}$.

El lector se habrá percatado de que, como Rousseau, Monboddo creía en la bonté naturelle del orangután; ese animal, aunque incapaz para la moralidad propiamente dicha, tiene, a fortiori, los verdaderos aspectos primitivos que nuestra especie tenía: una disposición "mansa" y "amable". Y de nuevo como Rousseau, Monboddo encuentra en esta conexión la ocasión para discrepar de la opinión de Hobbes:

Creo que nunca podría entender, como el Sr. Hobbes, que el hombre es por naturaleza el enemigo del hombre, y que el estado de naturaleza es un estado de guerra de cada hombre contra cada hombre. Ese es un estado como no existe ningún otro, ni existió jamás en especie alguna de animales. Y, aunque haya podido ser muy ingenioso por parte del Sr. Hobbes (pues es cierto que él fue un hombre muy perspicaz y mucho más erudito que los que actualmente se erigen como maestros de la filosofía), para mí está claro que no sabía lo que era el hombre por naturaleza, despojado de todos los hábitos y opiniones que adquiere en su vida civil; sino que suponía que, previamente a la institución de la sociedad, tenía todos los deseos y pasiones que ahora tiene ${ }^{23}$.

Esto obviamente implicaba que los deseos y ambiciones que hacen al hombre agresivo y lo colocan en desacuerdo con sus semejantes se desarrollaron a partir del instante en que adoptó el hábito de vivir en sociedad, y que sus pasiones antisociales son por tanto, en cierto sentido, producto del estado social. Esta idea juega un papel importante en el Second Discours de Rousseau. Como ya he señalado, mientras rechaza la psicología de Hobbes en la descripción del hombre en el estado de naturaleza, la acepta como cierta -y cada vez más cierta- para el hombre en la sociedad civilizada, de tal manera que el estadio que en Rousseau se aparta bastante de $s u$ "estado de naturaleza" se corresponde casi exactamente con el "estado de naturaleza" del filósofo de Malmesbury. Monboddo, sin embargo, no exagera y aporta una visión de la naturaleza humana en condiciones de vida social mucho menos desfavorable que la de Rousseau. 
¿Fue Monboddo, al contrario que Rousseau, un evolucionista en los sentidos tanto biológico como antropológico? Es decir, ¿aceptaba la hipótesis general sobre la transformación de las especies que había sido ya propuesta por Maupertuis y Diderot? ${ }^{24}$ En lo que respecta al tratado publicado por Monboddo, la respuesta al principio parecería negativa. Podría naturalmente considerarse que el empeño que pone en mostrar que el hombre y el orangután son de la misma especie implica que animales de diferentes especies no pueden surgir una de la otra o de antecesores comunes. Y en un pasaje niega expresamente que hubiera sugerido con anterioridad nuestro parentesco consanguíneo con los monos o con los otros simios:

Aunque mantengo que el orangután es de nuestra especie, no se debe suponer que crea que el mono o simio, con o sin cola, participa de nuestra naturaleza; al contrario, mantengo que, por mucho que su forma se parezca a la nuestra, éste es sin embargo, como Linneo dice del Troglodita, nec nostri generis, nec sanguinis ${ }^{25}$.

La razón principal que da para esto es que "ni el mono, ni el simio, ni el mandril tienen nada de manso o amable, tratable o dócil, benevolente o humano en su disposición; más bien al contrario, son maliciosos e intratables, sólo los gobierna la fuerza o el temor, y no tienen gravedad o compostura alguna en su modo de andar y en su comportamiento, tal como tiene el orangután". En consecuencia, esos rasgos del bandarlog que a algunos les parecía indicar su consanguinidad con la humanidad, son citados por Monboddo como evidencia de que no pueden estar relacionados con el orangután ni, por tanto, con nosotros.

Sin embargo, hay razones para pensar que su creencia real se inclinaba hacia la hipótesis más amplia rechazada por él mismo en el último pasaje citado. En una carta de junio de 1773 -esto es, muy poco después de la publicación del primer volumen de su libro-escribe:

Creo que es muy evidente que el orangután está por encima de la raza simia, a la cual creo que muy correctamente niegas la relación fraterna, aunque pienso que esa raza está emparentada con la nuestra, si bien que no relacionada tan directamente. Ya que me da la sensación de que los grandes mandriles se encuentran en la misma relación con nosotros que el asno con el caballo, o nuestro jilguero con el canario ${ }^{26}$.

Esto, aparentemente, solo puede significar que todos los simios, los monos y el hombre descienden de ancestros comunes. Como Monboddo no había clasificado a todos estos animales como pertenecientes a una sola especie, daba a entender que era

26 Knight, Lord Monboddo and His Contemporaries, p. 85. 
posible que una especie descendiera de otra. E incluso en Origin and Progress of Language la misma creencia era más que simplemente insinuada. Monboddo introduce en el trabajo algunos informes sobre la existencia en ciertas partes del mundo de hombres con cola ${ }^{27}$. Por ejemplo, un lugarteniente naval sueco cuya buena fe era garantizada por el propio Linneo había informado de que navegando por el golfo de Bengala, había "desembarcado en la costa de una de las islas de Nicobar, donde vieron hombres con colas como las de los gatos, y cuya manera de moverse era idéntica"28. Una historia similar había sido recogida por el gran Harvey. Sin embargo, no era necesario ir a lugares remotos de la tierra en busca de ejemplos. Monboddo ofrecía "la presentación de evidencia legal a través de testigos aún vivos" en referencia a un profesor de matemáticas en Inverness que "tenía una cola de medio pie de largo", que cuidadosamente ocultó durante su vida, "pero que fue descubierta después de su muerte, ocurrida hace alrededor de veinte años". Lo que es cierto, de cualquier modo, como Monboddo señala, es que todos tenemos colas rudimentarias, en forma de $o s$ coccygis. En la actualidad, los antropoides, como Monboddo ya sabía, no tienen colas, o al menos colas mucho más desarrolladas que las de los hombres, y sus historias de homines caudati, por tanto, no eran pertinentes en un argumento a favor de nuestro parentesco con el orangután. Él mismo afirma que cuenta "este hecho extraordinario relativo a nuestra especie como una curiosidad, aunque no pertenece a (su) asunto, excepto en cuanto que intenta ofrecernos una visión más amplia de la naturaleza humana". Pero la existencia ocasional de hombres con cola, y la presencia de vestigios de colas en el hombre y en el orangután, tiende a indicarnos que ambos son descendientes de ancestros remotos que estaban dotados de ese encantador y útil miembro. Y que Monboddo, con sus consideraciones, tenía la intención de sugerir esta hipótesis puede verse en una de sus notas a pie de página:

Aquellos que no han estudiado la variedad de la naturaleza en los animales, y particularmente en el hombre, el más variado de todos los animales, creerán que esta historia de hombres con cola es muy ridícula; y se reirán de la credulidad del autor porque parece creer esas historias. Pero el filósofo, que está más dispuesto a hacerse preguntas que a reír y a mofarse, no rechazará inmediatamente como algo increíble que pueda haber tal variedad en nuestra especie, al igual que en la tribu simia, que es pariente nuestra ${ }^{29}$.

27 La existencia de hombres con cola había sido asegurada por Plinio Nat. hist. vii. 2 y no fue rechazada por Linneo, Systema naturae (2ed., 1766), I, p. 33. Robinet había dedicado un capítulo de su Gradation naturelle des formes de l'être a la evidencia de la realidad de hombres con cola, que para él ilustraban lo finamente matizada que es la escala de los seres. El pongo (sin cola) "está conectado con el hombre por infinidad de similitudes; el hombre debe estar conectado por otras características con especies muy inferiores al pongo" (De la nature, V [1768], p. 160). Robinet, sin embargo, mantenía que el pongo y el orangután no eran "hombres verdaderos", sino "una especie intermedia que actúa como transición entre el simio y el hombre" (ibíd., p. 151). Robinet tiene un lugar junto con Maupertuis y Diderot entre los pioneros franceses del evolucionismo.

28 O. and P., I, p. 258.

29 Ibíd., p. 262. 
Ahora bien, "la tribu simia" quería decir, en la terminología de Monboddo, no los orangutanes, sino los monos, así que afirma aquí la probable verdad del punto de vista que en otra página parece negar. Se puede concluir, por tanto, que en principio aceptaba la posibilidad general de la transformación de las especies, y que definitivamente presentó, como hipótesis probable, el origen común de la mayor parte o de todos los antropoides. Se convirtió así (por lo que yo sé) en el primer británico defensor del evolucionismo, o cuasi-evolucionismo en biología, anticipándose en veinte años al Zoonomía de Erasmus Darwin ${ }^{30}$.

Monboddo, por supuesto, era suficientemente consciente del tipo de objeción sentimental que su hipótesis evocaría: la objeción contra el evolucionismo que un obispo de Oxford repitió en una famosa ocasión cerca de un siglo después. Ahora bien, Monboddo la sostuvo tenazmente, haciendo uso de la respuesta que el evolucionista contemporáneo emplea normalmente:

En cuanto a los vulgares, no puedo esperar que reconozcan parentesco alguno con aquellos habitantes de los bosques de Angola; sino que, por falso orgullo, continúen pensando que es altamente despectivo para la naturaleza humana lo que para el filósofo, por el contrario, es el elogio más grande del hombre: que desde el estado salvaje en el que el orangután vive haya sido capaz de llegar, por su propia sagacidad y esfuerzo, al estado en el que ahora lo vemos ${ }^{31}$.

\section{La diferencia específica del "homo sapiens"}

Aunque haya cabida para la disputa respecto a las diferencias entre el hombre y otros animales, había afirmado Rousseau en el Discurso sobre la desigualdad

hay una cualidad muy específica que los distingue, y sobre la cual no puede haber discrepancia alguna, y es la facultad de perfeccionarse; facultad que, con ayuda de las circunstancias, desarrolla sucesivamente todas las demás [...]; mientras que un animal es al cabo de unos pocos meses lo que será toda su vida, y su especie después de mil de años lo que fue el primer año de esos mil.

Monboddo recurre frecuentemente al mismo tema.

No hay ninguna diferencia natural entre nuestras mentes y las suyas (la de las bestias), y la superioridad que tenemos sobre ellas es adventicia [...]. Suponiendo que podamos llegar más lejos que donde pueda llegar con cultura

30 Akenside, sin embargo, había presagiado de un modo bastante oscuro la teoría de origen en Pleasure of the Imagination (1774), Lobro II; cfr. G: R: Potter en Mod. Phil., XXIV (1926), pp. 55-64, y Lovejoy, The Great Chain of Being, pp. 263-265.

31 O. and P., I, 360; cfr. también pp. 437-441. 
cualquier bestia (lo cual creo que ocurre), esto no es más que decir que [...] tenemos por Naturaleza capacidades mayores ${ }^{32}$.

Al hombre se le llama "animal racional", pero "esta diferencia específica de racional no consiste en la energía o el ejercicio actual de la facultad de la razón, ni siquiera en la posesión; en tal caso el recién nacido no sería un hombre". Y lo que es verdad sobre el individuo es verdad sobre la raza; la especie tenía al principio una pobre "capacidad de inteligencia y ciencia", y el tiempo demandó su desarrollo. Un escrito anónimo publicado antes de 1740 había anticipado el parecer de Monboddo y de Rousseau en este punto. El autor dice allí:

No puedo sino considerar algo defectuosa la común definición del hombre como animal racional. Creo que podía ser modificada favorablemente, aunque no completamente, si se le definiera como animal razonable, si se me permite que razonable signifique la capacidad de recibir razón, y no su ejercicio real [...]. Esta definición es apropiada, mientras que la de Aristóteles no lo es, ya que éste le define como animal racional, como si fuera efectivamente así, y no sólo potencialmente, por su naturaleza específica y sin ninguna ayuda exterior o cultura ${ }^{33}$.

\section{El ascenso del hombre}

Como consecuencia de todo lo anterior cabe decir que los atributos que comúnmente se consideran distintivos de la humanidad no fueron creados desde el principio, sino que se alcanzaron ardua y lentamente. Monboddo condensa felizmente la tesis más significativa de su doctrina en una adaptación de un verso de Virgilio: "Tantae molis erat humanam condere gentem". En resumen, nada que sea distintivo del hombre era primitivo, y nada que sea de excelencia máxima en él viene solo por naturaleza.

Monboddo fue por tanto un evolucionista en un sentido más profundo que el que se extrae de la creencia en la igualdad del hombre primitivo con el orangután. Fue uno de los pocos hombres de su tiempo que realmente tenía lo que podría llamarse "mente de hábito genético". La distinción aristotélica "entre el poder de llegar a ser

33 A Philosophical Dissertation upon the Inlets to Human Knowledge (reimpresión; Dublín, 1740), pp. 47 y 57. Cuarenta años después de Monboddo, Destutt de Tracy todavía enunciaba la misma doctrina con el entusiasmo del predicador de una nueva revelación: "Hemos recibido de esta admirable Naturaleza -es decir, de nuestra propia organización- sólo la posibilidad de perfeccionarnos a nosotros mismos. Cuando salimos de sus manos sólo poseíamos el germen de los medios para alcanzar el conocimiento [...]. De forma que somos enteramente obras de arte de nuestro propio trabajo; y tenemos tan poco parecido con el hombre de la naturaleza, con nuestro modo original de existencia, como un roble con una bellota o un ave con un huevo" (Élementes d'idéologie, 1817, cáp. XV, p. 289). 
algo, y la realidad de ser esa cosa", o "entre potencia y acto", es fundamental para toda su doctrina. Monboddo declara que

esta distinción recorre toda la naturaleza, en la cual hay un progreso perpetuo de un estado a otro, y que nada es al principio lo que llega a ser con el tiempo. Ahora bien, si alguien dice que la mente humana es una excepción a esta ley de la naturaleza, debe probarlo. Pero esto nunca le será posible ${ }^{34}$.

En consecuencia, Monboddo no vaciló en decir - por muy ofendidas que pudieran sentirse "algunas personas piadosas y bien dispuestas"- que "la principal prerrogativa de la naturaleza humana, el alma racional", es "de nuestra adquisición, y fruto del esfuerzo, como cualquier arte o ciencia, no un don de la naturaleza". Llámese a esta doctrina como se quiera, no puede adjudicársele el calificativo de "primitivismo". No obstante, incluso en nuestros días podemos encontrar autores eruditos que declaran que Monboddo era "un primitivista del tipo más extremo"35.

Aun más, este modo de pensar atacó al corazón del primitivismo y también del uniforme concepto de naturaleza humana con el que comúnmente se asociaba al primitivismo desde el siglo XVI al XVIII ${ }^{36}$. En realidad, esto ya estaba siendo minado por la "teoría de los climas", especialmente a través de la influencia de Montesquieu. Pero aun más serio fue el ataque llevado a cabo por estos tempranos evolucionistas sociales, Rousseau y Monboddo. En un comentario sobre las teorías de ciertos filósofos políticos, Montesquieu escribe:

Debo hacer una advertencia sobre cierta manera de razonar muy común que observo sobre este asunto. En primer lugar, se establece la hipótesis de que el hombre ha sido desde el principio, en todas las edades y naciones del mundo, el mismo, o casi el mismo, que el hombre actual de Europa o de otras partes civilizadas de la tierra, pues es una máxima constante en boca de tales razonadores que la naturaleza humana es y siempre ha sido la misma. Y en segundo lugar, tales razonadores suponen que esta máxima es innegable, de lo que concluyen, basándose en nuestros modos y costumbres y en que tal y tal institución son practicadas por las naciones civilizadas, que todo esto debe haber estado siempre en uso y que es tan antiguo como la raza humana [...]. Pero yo pienso que estoy en el derecho de establecer hipótesis contra hipótesis, y de suponer que el hombre, lejos de continuar siendo la misma criatura, ha variado más que ningún otro ser que conozcamos en la Naturaleza. Y aunque pueda decirse en algunos casos que su naturaleza es la misma, puesto que tiene todavía las mismas capacidades naturales que tenía desde el principio; sin embargo su naturaleza es, por su constitución original, susceptible de cambios mayores que la naturaleza de cualquier otro animal conocido. Y de hecho ha sido demostrado que ha sufrido los mayores cambios. Lo

$34 \quad$ O. and P., I, p. 438.

35 H. N. Fairchild, The Noble Savage, p. 331. Mr. Fairchild también señala, sin embargo, que Monboddo (incongruentemente) "anticipó la teoría de la evolución".

36 Sobre esto véase "The Parallel of Deism and Clasicism", citado más adelante. 
ha sido en primer lugar por la historia general de la humanidad, según la cual parece que ha habido un progreso gradual en las artes y los modos de varias naciones de la tierra [...]; y, en segundo lugar, por informes particulares sobre las costumbres y modos de las naciones bárbaras, tanto antiguas como modernas $^{37}$.

En este pasaje podemos ver uno de los presagios de esa desconfianza en las fórmulas universales, ese relativismo evolutivo peculiar en la filosofía política y social que llegaría a constituirse en uno de los principales rasgos distintivos del siglo XIX con respecto a los siglos modernos anteriores, aún imperfectamente asimilado por gran parte de la humanidad.

\section{Concepción de una historia universal evolutiva}

El gran proyecto original de Monboddo consistía en hacer a gran escala aquello que Rousseau había intentado de un modo breve y esquemático en el Discours sur l'origine de l'inégalité. En 1766 Monboddo transmitió a Harris su proyecto:

[Escribir] una Historia del Hombre en la cual seguiría la pista del hombre a través de los distintos estadios de su existencia; y es que nuestra especie ha progresado desde un estado algo superior al de la mera animalidad hasta el estado más perfecto de la antigua Grecia, de cuya descripción vos sois autor y que es tan sorprendente y peculiar de nuestra especie ${ }^{38}$.

Desgraciadamente no le quedó más remedio que abandonar este plan, encontrándolo "demasiado extenso para [sus] habilidades y para el tiempo que había de dedicarle". De resultas de ello, tan solo abordó una parte del programa original que consistía principalmente en una descripción del origen y la evolución del lenguaje. Sobre este tema ya más reducido logró escribir unas tres mil páginas en los descansos de sus deberes judiciales, dedicándose a ello a partir de los cincuenta años. No nos ocupan aquí sus especulaciones lingüísticas; lo que interesa es simplemente que, al igual que Rousseau, también él vislumbró un nuevo tipo de historia posible, e insistió en que una ciencia dedicada a tal asunto debía fundamentarse sobre un estudio cuidadoso de la vida real de los pueblos en los estadios anteriores de la evolución social:

Quienes han estudiado la historia del hombre, no sólo de naciones particulares, es decir, quienes han estudiado la historia desde la perspectiva amplia y liberal que distingue la naturaleza del hombre del hecho y la experiencia, saben muy bien que todas las naciones, incluso las más cultas y civilizadas sobre las cuales leemos en la historia, eran originalmente bárbaras [...]. Por 
tanto, quien quiera seguir la pista de la naturaleza del hombre hasta su origen, debe estudiar muy diligentemente las costumbres de las naciones bárbaras, y no formar teorías sobre el hombre a partir de lo que observa en las naciones civilizadas. Puede dudarse de que, por los descubrimientos hechos hasta ahora, podamos trazar de esta manera el recorrido del hombre hasta lo que supongo que ha sido su estado original, pero es cierto que podemos acercarnos mucho ${ }^{39}$.

Sobre la posibilidad de cumplir tal tarea en su propio tiempo, Monboddo fue indebidamente optimista. Sin embargo, expresó una concepción justa del programa a seguir, si cabe demasiado favorable para su logro real incluso en relación con la historia del lenguaje: "Mi sistema se fundamenta, no en la hipótesis, sino en la historia del hombre, recogida mediante hechos de manera idéntica a como recogemos la historia de cualquier otro animal" 40 .

Se puede decir con justicia que buena parte de la doctrina de Monboddo, y particularmente aquella parte más revolucionaria en su tiempo, era una elaboración de un grupo de ideas interrelacionadas que encontramos en el Discours sur l'origine de l'inégalité de Rousseau, publicado veinte años antes que el Origin and Progress of Language. ¿Se debía esta similitud a la ocurrencia espontánea de los mismos pensamientos en dos mentes coetáneas, o a la influencia directa de los primeros trabajos de un escritor sobre el otro? La cuestión no puede responderse con certeza. Ambos conocían por supuesto los relatos epicúreos sobre el hombre primitivo y sobre la evolución gradual de la sociedad; ambos conocían la obra de Lucrecio, Cicerón y Horacio, y a ninguno de los dos debió pasarles inadvertido el pasaje de las Sátiras de Horacio (i, 3, p. 99 y ss.) que Monboddo tomó como lema para su libro. Gran parte del Second Discours de Rousseau puede describirse como una ingeniosa combinación de su vena anti-primitivista de tradición clásica con su tendencia primitivista representada en dicha tradición, de diferentes formas, por Ovidio y Séneca ${ }^{41}$. Dado que Monboddo era ante todo un estudioso del mundo clásico y un entusiasta de la Antigüedad, es del todo posible que, como ha asumido Knight, sus ideas sobre estos temas le fueran sugeridos por primera vez en la lectura de autores antiguos ${ }^{42}$. Ambos conocían también los hechos revelados por el progreso de la anatomía comparativa en su siglo, y los dos eran fervientes lectores de descripciones, algunas de ellas recientes, de pueblos

O. and P., I, p. 145 .

Ibíd., p. 444.

Cfr. Primitivism and Related Ideas in Antiquity, pp. 43-49 y 263-286.

El mismo Monboddo escribe: "Sé que mi opinión sobre este asunto se considerará nueva y singular; pero sólo es una antigua opinión renacida pues, como he mostrado, ésta era la opinión de todos aquellos antiguos filósofos que se ocuparon del estado original del hombre antes de la sociedad y de la civilización" $(O$. and $P$., I). Los escritores clásicos citados para apoyar esto (ibíd., p. 368 ss.) son Horacio, Lucrecio, Platón (Laws, Libro i; Theatetus 186c; Timaeus 47a), Diodoro, Siculus, Cicerón. Cfr. también I, p. 298: "He intentado respaldar la antigua definición de hombre, y mostrar que ésta conviene al Orangután, aun cuando no tenga éste la facultad del habla". 
primitivos y de los simios antropoides. En particular, el número creciente de descripciones de aquellos salvajes nada nobles, los hotentotes, por parte de viajeros de finales del siglo XVII y del siglo XVIII tendían a sugerir firmemente una imagen desfavorable de la "condición original de la humanidad"43. Por otro lado, es cierto que Monboddo había leído el Discours de Rousseau antes de escribir su libro, que este último contiene un número de páginas muy similar a algunos de los de Rousseau, $\mathrm{y}$ que Monboddo fue de los más entusiastas admiradores que Rousseau encontró entre los de su generación.

Monboddo declara que "incluso los filósofos (con excepción de uno) no parecen saber nada de este estado" de naturaleza. Esta única excepción se identifica en una nota a pie de página en la que se dice: "El señor Rousseau, un gran genio, a mi juicio, pero al que se ha considerado extraño y caprichoso por haber dedicado tantos elogios al estado natural del hombre" 44 . Al insistir en lo indispensable de llevar a cabo un estudio sobre los salvajes del presente para poder conocer las condiciones primeras

43 La combinación de la casi universal concepción actual de la naturaleza como un continuo de formas (cadena de seres) con los hechos relatados sobre los hotentotes desde hacía tiempo, había llevado a algunos escritores de finales del siglo XVII y principios del xVIII a aproximarse, sin realmente anticiparla, a la doctrina propuesta por Rousseau y Monboddo. Cfr. la afirmación de Sir John Ovington, Voyage to Surat (1696), citada por R. W. Frantz en Modern Philology (XXVIII (1931), pp. 55-57): Los hotentotes son "exactamente lo contrario del género Humano [...], de modo que si existe un punto intermedio entre un Animal Racional y una Bestia, el Hotentote reivindica justamente este lugar". La teoría de Monboddo había sido bosquejada de un modo más próximo aun por Blackmore y Hughes en Lay Monastery: "Nada es más sorprendente y deleitoso que observar la escala o el gradual ascenso de los minerales a las plantas, de las plantas a los animales, y de los animales a la naturaleza humana. Es fácil distinguir estos tipos, hasta alcanzar al más elevado de uno, y al más bajo del que está por encima de él; y entonces la diferencia es tan agradable, que los límites y las fronteras de sus especies parecen haber sido dejadas sin establecer por la Naturaleza para perplejidad del curioso y humillación del filósofo orgulloso. Así como el hombre, quien más se acerca a la clase más baja de los espíritus celestiales (ya que ciertamente debemos suponer una subordinación en ese orden celestial) al ser mitad cuerpo mitad espíritu, se convierte en el aequator que divide por la mitad la creación entera y distingue lo corporal del mundo intelectual invisible; del mismo modo el simio o mono, que disfruta de la mayor similitud con el hombre, es el siguiente en el orden de los animales por debajo de él. Tampoco es tan grande la disconformidad entre los individuos más bajos de nuestra especie y el simio o mono, y si estos últimos estuvieran dotados de la facultad del habla, quizás podrían reclamar justamente el rango y la dignidad de la raza humana, como el salvaje hotentote, o el estúpido nativo de Nueva Zelanda [...]. El más perfecto en este orden de seres, el orangután, como lo llaman los nativos de Angola, es decir, el hombre salvaje, u hombre de los bosques, tiene el honor de disfrutar del mayor parecido con la naturaleza humana. Aunque toda esa especie tiene cierta consonancia con nosotros en nuestros rasgos, pues se han encontrado muchos ejemplos de hombres con cara de mono, éste tiene de entre ellos el mayor parecido, no sólo en su semblante, sino en la estructura de su cuerpo, y en su habilidad para caminar erguido, así como a cuatro patas, en sus órganos del habla, en su pronta comprensión, y en sus amables y tiernas pasiones, cualidades que no se encuentran en ningún otro tipo de simio, y algunos otros rasgos" ( $N^{\circ} .5$ [1714], p. 28. Nueva edición de Lay Monk [1713]).

$44 \quad$ O. and P., I, III. Monboddo era por supuesto bien consciente de que Rousseau no había representado "el estado natural del hombre" como el ideal en su conjunto. 
de toda la humanidad en lugar de intentar "formar un sistema de naturaleza humana basado solo en la observación de las naciones civilizadas", Monboddo vuelve a referirse al señor Rousseau, particularmente a su tratado sobre l'inégalité parmi les hommes, donde ridiculiza la locura de aquellos que piensan que comprenden la naturaleza humana porque conocen los modos y maneras de los de su nación, y quizá algunos de los de las naciones vecinas; de lo que concluyen muy sabiamente que el hombre es el mismo en todas las edades y en todas las naciones". "Me siento feliz", añade Monboddo, "al ver que mis ideas, tanto respecto al estado original de la naturaleza humana, como al origen del lenguaje, están tan perfectamente de acuerdo con las nociones de un autor de tal genio, pensamiento original y erudición"45. Sin embargo, Monboddo no dice que derivara su teoría de la humanidad del orangután de Rousseau, y más bien da a entender que dieron con la gran idea de manera independiente.

El señor Rousseau, en su obra antes citada, nota 10 , ha recogido algunos informes aportados por los viajeros sobre este animal, y parece que coincidimos en la opinión de que pertenece a nuestra especie, rechazando firmemente la idea de los que piensan que el habla es natural al hombre. Ahora bien, si superamos ese prejuicio, y no insistimos en que otras artes de la vida, de las que los orangutanes carecen, son del mismo modo naturales al hombre, es imposible que podamos rechazar el darles el apelativo de hombres ${ }^{46}$.

Todo lo que se puede afirmar con confianza sobre el tema es que Rousseau y Monboddo eran compañeros de batalla, los dos principales campeones de su época en la defensa de las seis tesis conectadas que he presentado al comienzo de este trabajo, y que la prioridad de Rousseau en el enunciado de todas ellas ciertamente pone en cuestión la originalidad de Monboddo en estos puntos. No obstante, este último las desarrolló mucho más ampliamente. La mayor parte de los británicos educados en los años ochenta [del siglo XVIII] lo consideraron un autor original quien, de un modo algo indeciso, habría extendido la doctrina de Rousseau sobre la igualdad de las especies del hombre y el chimpancé hacia la hipótesis del origen común de todos los antropoides, y quien habría sugerido en consecuencia una ley general sobre la evolución orgánica. En esta última tesis se le anticiparon al menos tres escritores franceses (Maupertuis, Diderot, Robinet) y Leibniz; pero aparentemente no era consciente de ello, como la mayor parte de sus contemporáneos británicos y como muchas historias de la ciencia durante más de un siglo. No es sorprendente, por tanto, que un hombre de campo haya reclamado para él -y por tanto para Escocia- el crédito normalmente atribuido al autor del Origen de las Especies:

Aunque Darwin proclama la ley, Y la extiende por el mundo, jay!

45 Ibíd., p. 152; cfr. p. 381: "ese genio singular que nuestra era ha producido, el Sr. Rousseau".

$46 \quad O$. and P., I, p. 189. 
El hombre que antes vio el secreto

Fue Monboddo, anciano y honesto.

El arquitecto tiene precedencia

Sobre quien lleva la espuerta, ¡ay!

Alcémonos contra ellos, Pueblo Recio,

A Monboddo reivindiquemos. 